\title{
On nonlinear stability in various random normed spaces
}

\author{
John Michael Rassias ${ }^{1}$, Reza Saadati ${ }^{*}$, Ghadir Sadeghi $^{3}$ and J Vahidi ${ }^{4}$
}

\footnotetext{
* Correspondence: RSAADATI@EML. $\mathrm{CC}$

2Department of Mathematics, Science and Research Branch, Islamic Azad University, Tehran, Iran Full list of author information is available at the end of the article
}

$$
\begin{aligned}
& \text { Abstract } \\
& 16 f(x+4 y)+f(4 x-y)=306\left[9 f\left(x+\frac{y}{3}\right)+f(x+2 y)\right] \\
& +136 f(x-y)-1394 f(x+y)+425 f(y)-1530 f(x)
\end{aligned}
$$

in the setting of random normed spaces Furthermore, the interdisciplinary relation among the theory of random spaces, the theory of non-Archimedean space, the theory of fixed point theory, the theory of intuitionistic spaces and the theory of functional equations are also presented in the article.

Keywords: generalized Hyers-Ulam stability, quartic functional equation, random normed space, intuitionistic random normed space

\section{Introduction}

The study of stability problems for functional equations is related to a question of Ulam [1] concerning the stability of group homomorphisms and affirmatively answered for Banach spaces by Hyers [2]. Subsequently, this result of Hyers was generalized by Aoki [3] for additive mappings and by Rassias [4] for linear mappings by considering an unbounded Cauchy difference. The article of Rassias [4] has provided a lot of influence in the development of what we now call generalized Ulam-Hyers stability of functional equations. We refer the interested readers for more information on such problems to the article [5-17].

Recently, Alsina [18], Chang, et al. [19], Mirmostafaee et al. [20], [21], Miheț and Radu [22], Miheț et al. [23], [24], [25], [26], Baktash et al. [27], Eshaghi et al. [28], Saadati et al. [29], [30] investigated the stability in the settings of fuzzy, probabilistic, and random normed spaces.

In this article, we study the stability of the following functional equation

$$
\begin{aligned}
16 f(x+4 y)+f(4 x-y)= & 306\left[9 f\left(x+\frac{y}{3}\right)+f(x+2 y)\right] \\
& +136 f(x-y)-1394 f(x+y)+425 f(y)-1530 f(x)
\end{aligned}
$$

in the various random normed spaces via different methods. Since $a x^{4}$ is a solution of above functional equation, we say it quartic functional equation.

\section{Springer}

(C) 2011 Rassias et al; licensee Springer. This is an Open Access article distributed under the terms of the Creative Commons Attribution License (http://creativecommons.org/licenses/by/2.0), which permits unrestricted use, distribution, and reproduction in any medium, provided the original work is properly cited. 


\section{Preliminaries}

In this section, we recall some definitions and results which will be used later on in the article.

A triangular norm (shorter t-norm) is a binary operation on the unit interval $[0,1]$, i.e., a function $T:[0,1] \times[0,1] \rightarrow[0,1]$ such that for all $a, b, c \in[0,1]$ the following four axioms satisfied:

(i) $T(a, b)=T(b, a)$ (commutativity);

(ii) $T(a,(T(b, c)))=T(T(a, b), c)$ (associativity);

(iii) $T(a, 1)=a$ (boundary condition);

(iv) $T(a, b) \leq T(a, c)$ whenever $b \leq c$ (monotonicity).

Basic examples are the Lukasiewicz $t$-norm $T_{L}, T_{L}(a, b)=\max (a+b-1,0) \forall a, b \in$ $[0,1]$ and the $t$-norms $T_{P}, T_{M}, T_{D}$, where $T_{P}(a, b):=a b, T_{M}(a, b):=\min \{a, b\}$,

$$
T_{D}(a, b):= \begin{cases}\min (a, b), & \text { if } \max (a, b)=1 \\ 0, & \text { otherwise }\end{cases}
$$

If $T$ is a $t$-norm then $x_{T}^{(n)}$ is defined for every $x \in[0,1]$ and $n \in N \cup\{0\}$ by 1 , if $n=$ 0 and $T\left(x_{T}^{(n-1)}, x\right)$, if $n \geq 1$. A $t$-norm $T$ is said to be of Hadžić-type (we denote by $T \in \mathcal{H}$ ) if the family $\left(x_{T}^{(n)}\right)_{n \in N}$ is equicontinuous at $x=1$ (cf. [31]).

Other important triangular norms are (see [32]):

-the Sugeno-Weber family $\left\{T_{\lambda}^{S W}\right\}_{\lambda \in[-1, \infty]}$ is defined by $T_{-1}^{S W}=T_{D}, T_{\infty}^{S W}=T_{P}$ and

$$
T_{\lambda}^{S W}(x, y)=\max \left(0, \frac{x+y-1+\lambda x y}{1+\lambda}\right)
$$

if $\lambda \in(-1, \infty)$.

-the Domby family $\left\{T_{\lambda}^{D}\right\}_{\lambda \in[0, \infty}$, defined by $T_{D}$, if $\lambda=0, T_{M}$, if $\lambda=\infty$ and

$$
T_{\lambda}^{D}(x, y)=\frac{1}{1+\left(\left(\frac{1-x}{x}\right)^{\lambda}+\left(\frac{1-y}{y}\right)^{\lambda}\right)^{1 / \lambda}}
$$

if $\lambda \in(0, \infty)$.

-the Aczel-Alsina family $\left\{T_{\lambda}^{A A}\right\}_{\lambda \in[0, \infty}$, defined by $T_{D}$, if $\lambda=0, T_{M}$, if $\lambda=\infty$ and

$$
T_{\lambda}^{A A}(x, y)=e^{-\left(|\log x|^{\lambda}+|\log y|^{\lambda}\right)^{1 / \lambda}}
$$

if $\lambda \in(0, \infty)$.

A $t$-norm $T$ can be extended (by associativity) in a unique way to an $\mathrm{n}$-array operation taking for $\left(x_{1}, \ldots, x_{n}\right) \in[0,1]^{n}$ the value $T\left(x_{1}, \ldots, x_{n}\right)$ defined by

$$
\mathrm{T}_{i=1}^{0} x_{i}=1, \mathrm{~T}_{i=1}^{n} x_{i}=T\left(\mathrm{~T}_{i=1}^{n-1} x_{i}, x_{n}\right)=T\left(x_{1}, \ldots, x_{n}\right) .
$$

$T$ can also be extended to a countable operation taking for any sequence $\left(x_{n}\right)_{n \in N}$ in $[0,1]$ the value

$$
\mathrm{T}_{i=1}^{\infty} x_{i}=\lim _{n \rightarrow \infty} \mathrm{T}_{i=1}^{n} x_{i} .
$$


The limit on the right side of (2.1) exists since the sequence $\left\{\mathrm{T}_{i=1}^{n} x_{i}\right\}_{n \in \mathbb{N}}$ is nonincreasing and bounded from below.

Proposition 2.1. [32] (i) For $T \geq T_{L}$ the following implication holds:

$$
\lim _{n \rightarrow \infty} \mathrm{T}_{i=1}^{\infty} x_{n+i}=1 \Leftrightarrow \sum_{n=1}^{\infty}\left(1-x_{n}\right)<\infty .
$$

(ii) If $T$ is of Hadžić-type then

$$
\lim _{n \rightarrow \infty} \mathrm{T}_{i=1}^{\infty} x_{n+i}=1
$$

for every sequence $\left\{x_{n}\right\}_{n \in N}$ in $[0,1]$ such that $\lim _{n \rightarrow \infty} x_{n}=1$.

(iii) If $T \in\left\{T_{\lambda}^{A A}\right\}_{\lambda \in(0, \infty)} \cup\left\{T_{\lambda}^{D}\right\}_{\lambda \in(0, \infty)}$, then

$$
\lim _{n \rightarrow \infty} \mathrm{T}_{i=1}^{\infty} x_{n+i}=1 \Leftrightarrow \sum_{n=1}^{\infty}\left(1-x_{n}\right)^{\alpha}<\infty .
$$

(iv) If $T \in\left\{T_{\lambda}^{S W}\right\}_{\lambda \in[-1, \infty)}$, then

$$
\lim _{n \rightarrow \infty} \mathrm{T}_{i=1}^{\infty} x_{n+i}=1 \Leftrightarrow \sum_{n=1}^{\infty}\left(1-x_{n}\right)<\infty .
$$

Definition 2.2. [33] A random normed space (briefly, $\mathrm{RN}$-space) is a triple $(X, \mu, T)$, where $X$ is a vector space, $T$ is a continuous $t$-norm, and $\mu$ is a mapping from $X$ into $D^{+}$such that, the following conditions hold:

(RN1) $\mu_{x}(t)=\varepsilon_{0}(t)$ for all $t>0$ if and only if $x=0$;

(RN2) $\mu_{\alpha x}(t)=\mu_{x}\left(\frac{t}{|\alpha|}\right)$ for all $x \in X, \alpha \neq 0$;

(RN3) $\mu_{x+y}(t+s) \geq T\left(\mu_{x}(t), \mu_{y}(s)\right)$ for all $x, y, z \in X$ and $t, s \geq 0$.

Definition 2.3. Let $(X, \mu, T)$ be an RN-space.

(1) A sequence $\left\{x_{n}\right\}$ in $X$ is said to be convergent to $x$ in $X$ if, for every $\varepsilon>0$ and $\lambda>0$, there exists a positive integer $N$ such that $\mu_{x_{n}-x}(\varepsilon)>1-\lambda$ whenever $n \geq N$.

(2) A sequence $\left\{x_{n}\right\}$ in $X$ is called Cauchy if, for every $\varepsilon>0$ and $\lambda>0$, there exists a positive integer $N$ such that $\mu_{x_{n}-x_{m}}(\varepsilon)>1-\lambda$ whenever $n \geq m \geq N$.

(3) An RN-space $(X, \mu, T)$ is said to be complete if every Cauchy sequence in $X$ is convergent to a point in $X$.

Theorem 2.4. [34] If $(X, \mu, T)$ is an RN-space and $\left\{x_{n}\right\}$ is a sequence such that $x_{n} \rightarrow x$, then $\lim _{n \rightarrow \infty} \mu_{x_{n}}(t)=\mu_{x}(t)$ almost everywhere.

\section{Non-Archimedean random normed space}

By a non-Archimedean field we mean a field $\mathcal{K}$ equipped with a function (valuation) $|\cdot|$ from $K$ into $[0, \infty]$ such that $|r|=0$ if and only if $r=0,|r s|=|r||s|$, and $|r+s| \leq \max \{\mid$ $r|| s \mid$,$\} for all r, s \in \mathcal{K}$. Clearly $|1|=|-1|=1$ and $|n| \leq 1$ for all $n \in \mathbb{N}$. By the trivial valuation we mean the mapping $|\cdot|$ taking everything but 0 into 1 and $|0|=0$. Let $\mathcal{X}$ be a vector space over a field $\mathcal{K}$ with a non-Archimedean non-trivial valuation $|\cdot|$. 
A function $\|\cdot\|: \mathcal{X} \rightarrow[0, \infty]$ is called a non-Archimedean norm if it satisfies the following conditions:

(i) $\|x\|=0$ if and only if $x=0$;

(ii) for any $r \in \mathcal{K}, x \in \mathcal{X},\|r x\|=\|r\||| x \|$;

(iii) the strong triangle inequality (ultrametric); namely,

$$
\|x+y\| \leq \max \{\|x\|,\|y\|\} \quad(x, y \in \mathcal{X}) .
$$

Then $(\mathcal{X},\|\cdot\|)$ is called a non-Archimedean normed space. Due to the fact that

$$
\left\|x_{n}-x_{m}\right\| \leq \max \left\{\left\|x_{j+1}-x_{j}\right\|: m \leq j \leq n-1\right\} \quad(n>m),
$$

a sequence $\left\{x_{n}\right\}$ is Cauchy if and only if $\left\{x_{n+1}-x_{n}\right\}$ converges to zero in a nonArchimedean normed space. By a complete non-Archimedean normed space we mean one in which every Cauchy sequence is convergent.

In 1897, Hensel [35] discovered the $p$-adic numbers as a number theoretical analogue of power series in complex analysis. Fix a prime number $p$. For any non-zero rational number $x$, there exists a unique integer $n_{x} \in \mathbb{Z}$ such that $x=\frac{a}{b} p^{n_{x}}$, where $a$ and $b$ are integers not divisible by $p$. Then $|x|_{p}:=p^{-n_{x}}$ defines a non-Archimedean norm on $Q$. The completion of $Q$ with respect to the metric $d(x, y)=|x-y|_{p}$ is denoted by $Q_{p}$, which is called the $p$-adic number field.

Throughout the article, we assume that $\mathcal{X}$ is a vector space and $\mathcal{Y}$ is a complete nonArchimedean normed space.

Definition 3.1. A non-Archimedean random normed space (briefly, non-Archimedean $\mathrm{RN}$-space) is a triple $(\mathcal{X}, \mu, T)$, where $X$ is a linear space over a non-Archimedean field $\mathcal{K}, T$ is a continuous $t$-norm, and $\mu$ is a mapping from $X$ into $D^{+}$such that the following conditions hold:

(NA-RN1) $\mu_{x}(t)=\varepsilon_{0}(t)$ for all $t>0$ if and only if $x=0$;

(NA-RN2) $\mu_{\alpha x}(t)=\mu_{x}\left(\frac{t}{|\alpha|}\right)$ for all $x \in \mathcal{X}, t>0, \alpha \neq 0$;

(NA-RN3) $\mu_{x+y}(\max \{t, s\}) \geq T\left(\mu_{x}(t), \mu_{y}(s)\right)$ for all $x, y, z \in \mathcal{X}$ and $t, s \geq 0$.

It is easy to see that if (NA-RN3) holds then so is

(RN3) $\mu_{x+y}(t+s) \geq T\left(\mu_{x}(t), \mu_{y}(s)\right)$.

As a classical example, if $(\mathcal{X},\|\|$.$) is a non-Archimedean normed linear space, then$ the triple $\left(\mathcal{X}, \mu, T_{M}\right)$, where

$$
\mu_{x}(t)= \begin{cases}0 & t \leq\|x\| \\ 1 & t>\|x\|\end{cases}
$$

is a non-Archimedean RN-space.

Example 3.2. Let $(\mathcal{X},\|\|$.$) be is a non-Archimedean normed linear space. Define$

$$
\mu_{x}(t)=\frac{t}{t+\|x\|}, \quad \forall x \in \mathcal{X} \quad t>0 .
$$

Then $\left(\mathcal{X}, \mu, T_{M}\right)$ is a non-Archimedean RN-space.

Definition 3.3. Let $(\mathcal{X}, \mu, T)$ be a non-Archimedean RN-space. Let $\left\{x_{n}\right\}$ be a sequence in $\mathcal{X}$. Then $\left\{x_{n}\right\}$ is said to be convergent if there exists $x \in \mathcal{X}$ such that 


$$
\lim _{n \rightarrow \infty} \mu_{x_{n}-x}(t)=1
$$

for all $t>0$. In that case, $x$ is called the limit of the sequence $\left\{x_{n}\right\}$.

A sequence $\left\{x_{n}\right\}$ in $\mathcal{X}$ is called Cauchy if for each $\varepsilon>0$ and each $t>0$ there exists $n_{0}$ such that for all $n \geq n_{0}$ and all $p>0$ we have $\mu_{x_{n+p}-x_{n}}(t)>1-\varepsilon$.

If each Cauchy sequence is convergent, then the random norm is said to be complete and the non-Archimedean $\mathrm{RN}$-space is called a non-Archimedean random Banach space.

Remark 3.4. [36] Let $\left(\mathcal{X}, \mu, T_{M}\right)$ be a non-Archimedean RN-space, then

$$
\mu_{x_{n+p}-x_{n}}(t) \geq \min \left\{\mu_{x_{n+j+1}-x_{n+j}}(t): j=0,1,2, \ldots, p-1\right\}
$$

So, the sequence $\left\{x_{n}\right\}$ is Cauchy if for each $\varepsilon>0$ and $t>0$ there exists $n_{0}$ such that for all $n \geq n_{0}$ we have

$$
\mu_{x_{n+1}-x_{n}}(t)>1-\varepsilon .
$$

\section{Generalized Ulam-Hyers stability for a quartic functional equation in non- Archimedean RN-spaces}

Let $\mathcal{K}$ be a non-Archimedean field, $\mathcal{X}$ a vector space over $\mathcal{K}$ and let $(\mathcal{Y}, \mu, T)$ be a nonArchimedean random Banach space over $\mathcal{K}$.

We investigate the stability of the quartic functional equation

$$
\begin{aligned}
16 f(x+4 y)+f(4 x-y) & =306\left[9 f\left(x+\frac{y}{3}\right)+f(x+2 y)\right] \\
& +136 f(x-y)-1394 f(x+y)+425 f(y)-1530 f(x),
\end{aligned}
$$

where $f$ is a mapping from $\mathcal{X}$ to $\mathcal{Y}$ and $f(0)=0$.

Next, we define a random approximately quartic mapping. Let $\Psi$ be a distribution function on $\mathcal{X} \times \mathcal{X} \times[0, \infty]$ such that $\Psi(x, y, \cdot)$ is symmetric, nondecreasing and

$$
\Psi(c x, c x, t) \geq \Psi\left(x, x, \frac{t}{|c|}\right) \quad(x \in \mathcal{X}, \quad c \neq 0) .
$$

Definition 4.1. A mapping $f: \mathcal{X} \rightarrow \mathcal{Y}$ is said to be $\Psi$-approximately quartic if

$$
\begin{aligned}
& \mu_{16 f(x+4 y)+f(4 x-y)-306\left[9 f\left(x+\frac{y}{3}\right)+f(x+2 y)\right]-136 f(x-y)+1394 f(x+y)-425 f(y)+1530 f(x)}(t) \\
& \quad \geq \Psi(x, y, t) \quad(x, y \in \mathcal{X}, \quad t>0) .
\end{aligned}
$$

In this section, we assume that $4 \neq 0$ in $\mathcal{K}$ (i.e., characteristic of $\mathcal{K}$ is not 4 ). Our main result, in this section, is the following:

Theorem 4.2. Let Kbe a non-Archimedean field, $\mathcal{X}$ a vector space over $\mathcal{K}$ and let $(\mathcal{Y}, \mu, T)$ be a non-Archimedean random Banach space over $\mathcal{K}$. Let $f: \mathcal{X} \rightarrow \mathcal{Y}$ be a $\Psi$ approximately quartic mapping. If for some $\alpha \in \mathbb{R}, \alpha>0$, and some integer $k, k>3$ with $\left|4^{k}\right|<\alpha$,

$$
\Psi\left(4^{-k} x, 4^{-k} y, t\right) \geq \Psi(x, y, \alpha t) \quad(x \in \mathcal{X}, \quad t>0)
$$

and

$$
\lim _{n \rightarrow \infty} \mathrm{T}_{j=n}^{\infty} M\left(x, \frac{\alpha^{j} t}{|4|^{k j}}\right)=1 \quad(x \in \mathcal{X}, \quad t>0),
$$


then there exists a unique quartic mapping $Q: \mathcal{X} \rightarrow \mathcal{Y}$ such that

$$
\mu_{f(x)-Q(x)}(t) \geq \mathrm{T}_{i=1}^{\infty} M\left(x, \frac{\alpha^{i+1} t}{|4|^{k i}}\right)
$$

for all $x \in X$ and $t>0$, where

$$
M(x, t):=T\left(\Psi(x, 0, t), \Psi(4 x, 0, t), \cdots, \Psi\left(4^{k-1} x, 0, t\right)\right) \quad(x \in \mathcal{X}, \quad t>0) .
$$

Proof. First, we show by induction on $j$ that for each $x \in \mathcal{X}, t>0$ and $j \geq 1$,

$$
\mu_{f\left(4^{j} x\right)-25 \sigma^{j} f(x)}(t) \geq M_{j}(x, t):=T\left(\Psi(x, 0, t), \cdots, \Psi\left(4^{j-1} x, 0, t\right)\right) .
$$

Putting $y=0$ in (4.1), we obtain

$$
\mu_{f(4 x)-256 f(x)}(t) \geq \Psi(x, 0, t) \quad(x \in \mathcal{X}, \quad t>0) .
$$

This proves (4.5) for $j=1$. Assume that (4.5) holds for some $j \geq 1$. Replacing $y$ by 0 and $x$ by $4^{j} x$ in (4.1), we get

$$
\mu_{f\left(4^{j+1} x\right)-256 f\left(4^{j} x\right)}(t) \geq \Psi\left(4^{j} x, 0, t\right) \quad(x \in \mathcal{X}, \quad t>0) .
$$

Since $|256| \leq 1$,

$$
\begin{aligned}
\mu_{f\left(4^{j+1} x\right)-256^{j+1} f(x)}(t) & \geq T\left(\mu_{f\left(4^{j+1} x\right)-256 f\left(4^{j} x\right)}(t), \mu_{256 f\left(4^{j} x\right)-256^{j+1} f(x)}(t)\right) \\
& =T\left(\mu_{f\left(4^{j+1} x\right)-256 f\left(4^{j} x\right)}(t), \mu_{f\left(4^{j} x\right)-256^{j} f(x)}\left(\frac{t}{|256|}\right)\right) \\
& \geq T\left(\mu_{f\left(4^{j+1} x\right)-256 f\left(4^{j} x\right)}(t), \mu_{f\left(4^{j} x\right)-25 \sigma^{j} f(x)}(t)\right) \\
& \geq T\left(\Psi\left(4^{j} x, 0, t\right), M_{j}(x, t)\right) \\
& =M_{j+1}(x, t)
\end{aligned}
$$

for all $x \in \mathcal{X}$. Thus (4.5) holds for all $j \geq 1$. In particular

$$
\mu_{f\left(4^{k} x\right)-256^{k} f(x)}(t) \geq M(x, t) \quad(x \in \mathcal{X}, \quad t>0) .
$$

Replacing $x$ by $4^{-(k n+k)} x$ in (4.6) and using inequality (4.2), we obtain

$$
\begin{aligned}
\mu_{f\left(\frac{x}{4^{k n}}\right)-256^{k} f\left(\frac{x}{4^{k n+k}}\right)}(t) & \geq M\left(\frac{x}{4^{k n+k}}, t\right) \\
& \geq M\left(x, \alpha^{n+1} t\right) \quad(x \in \mathcal{X}, \quad t>0, \quad n=0,1,2, \ldots) .
\end{aligned}
$$

Then

$$
\mu_{\left(4^{4 k}\right)^{n} f\left(\frac{x}{\left(4^{k}\right)^{n}}\right)-\left(4^{4 k}\right)^{n+1} f\left(\frac{x}{\left(4^{k}\right)^{n+1}}\right)}(t) \geq M\left(x, \frac{\alpha^{n+1}}{\left|\left(4^{4 k}\right)^{n}\right|} t\right) \quad(x \in \mathcal{X}, \quad t>0, \quad n=0,1,2, \ldots) .
$$

Hence,

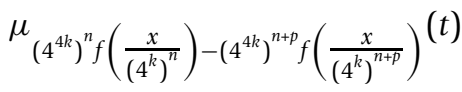

$$
\begin{aligned}
& \geq T_{j=n}^{n+p}\left(\mu_{\left(4^{4 k}\right)^{j} f\left(\frac{x}{\left(4^{k}\right)^{j}}\right)-\left(4^{4 k}\right)^{j+p} f\left(\frac{x}{\left(4^{k}\right)^{j+p}}\right)}(t)\right) \\
& \geq T_{j=n}^{n+p} M\left(x, \frac{\alpha^{j+1}}{\left|\left(4^{4 h}\right)^{j}\right|} t\right) \\
& \geq T_{j=n}^{n+p} M\left(x, \frac{\alpha^{j+1}}{\left|\left(4^{k}\right)^{j}\right|} t\right) \quad(x \in \mathcal{X}, \quad t>0, \quad n=0,1,2, \ldots) .
\end{aligned}
$$


Since $\lim _{n \rightarrow \infty} T_{j=n}^{\infty} M\left(x, \frac{\alpha^{j+1}}{\left|\left(4^{k}\right)^{j}\right|} t\right)=1 \quad(x \in \mathcal{X}, \quad t>0),\left\{\left(4^{4 k}\right)^{n} f\left(\frac{x}{\left(4^{k}\right)^{n}}\right)\right\}_{n \in N}$, is a Cauchy sequence in the non-Archimedean random Banach space $(\mathcal{Y}, \mu, T)$. Hence, we can define a mapping $Q: \mathcal{X} \rightarrow \mathcal{Y}$ such that

$$
\lim _{n \rightarrow \infty} \mu_{\left(4^{4 k}\right)^{n} f\left(\frac{x}{\left(4^{k}\right)^{n}}\right)-Q(x)}(t)=1 \quad(x \in X, \quad t>0) .
$$

Next, for each $n \geq 1, x \in \mathcal{X}$ and $t>0$,

$$
\begin{aligned}
& \mu_{f(x)-\left(4^{4 k}\right)^{n} f\left(\frac{x}{\left(4^{k}\right)^{n}}\right)}(t)=\mu \sum_{i=0}^{n-1}\left(4^{4 k}\right)^{i} f\left(\frac{x}{\left(4^{k}\right)^{i}}\right)-\left(4^{4 k}\right)^{i+1} f\left(\frac{x}{\left(4^{k}\right)^{i+1}}\right)(t)
\end{aligned}
$$

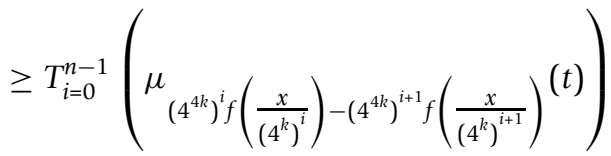

$$
\begin{aligned}
& \geq T_{i=0}^{n-1} M\left(x, \frac{\alpha^{i+1} t}{\left|4^{4 k}\right|^{i}}\right) \text {. }
\end{aligned}
$$

Therefore,

$$
\begin{aligned}
\mu_{f(x)-Q(x)}(t) & \geq T\left(\mu_{f(x)-\left(4^{4 k}\right)^{n} f\left(\frac{x}{\left(4^{k}\right)^{n}}\right)}(t), \mu_{\left(4^{4 k}\right)^{n} f\left(\frac{x}{\left(4^{k}\right)^{n}}\right)-Q(x)}(t)\right) \\
& \geq T\left(T_{i=0}^{n-1} M\left(x, \frac{\alpha^{i+1} t}{\left|4^{4 k}\right|^{i}}\right), \mu_{\left(4^{4 k}\right)^{n} f\left(\frac{x}{\left(4^{k}\right)^{n}}\right)-Q(x)}(t)\right) .
\end{aligned}
$$

By letting $n \rightarrow \infty$, we obtain

$$
\mu_{f(x)-Q(x)}(t) \geq T_{i=1}^{\infty} M\left(x, \frac{\alpha^{i+1} t}{\left|4^{k}\right|^{i}}\right) .
$$

This proves (4.4).

As $T$ is continuous, from a well-known result in probabilistic metric space (see e.g., [[34], Chapter 12]), it follows that

$$
\begin{aligned}
& \lim _{n \rightarrow \infty} \mu\left(4^{k}\right)^{n} \cdot 16 f\left(4^{-k n}(x+4 y)\right)+\left(4^{k}\right)^{n} f\left(4^{-k n}(4 x-y)\right)-306\left[\left(4^{k}\right)^{n} \cdot 9 f\left(4^{-k n}\left(x+\frac{y}{3}\right)\right)+\left(4^{k}\right)^{n} f\left(4^{-k n}(x+2 y)\right)\right] \\
& -136\left(4^{k}\right)^{n} f\left(4^{-k n}(x-\gamma)\right)+1394\left(4^{k}\right)^{n} f\left(4^{-k n}(x+y)\right)-425\left(4^{k}\right)^{n} f\left(4^{-k n} \gamma\right)+1530\left(4^{k}\right)^{n} f\left(4^{-k n} x\right)(t) \\
& =\mu_{16 Q(x+4 y)+Q(4 x-y)-306\left[9 Q\left(x+\frac{y}{3}\right)+Q(x+2 y)\right]-136 Q(x-y)+1394 Q(x+y)-425 Q(y)+1530 Q(x)}(t)
\end{aligned}
$$

for almost all $t>0$.

On the other hand, replacing $x, y$ by $4^{-k n} x, 4^{-k n} y$, respectively, in (4.1) and using (NARN2) and (4.2), we get

$$
\begin{aligned}
& \mu \\
& \left(4^{k}\right)^{n} \cdot 16 f\left(4^{-k n}(x+4 y)\right)+\left(4^{k}\right)^{n} f\left(4^{-k n}(4 x-y)\right)-306\left[\left(4^{k}\right)^{n} \cdot 9 f\left(4^{-k n}\left(x+\frac{y}{3}\right)\right)+\left(4^{k}\right)^{n} f\left(4^{-k n}(x+2 y)\right)\right] \\
& -136\left(4^{k}\right)^{n} f\left(4^{-k n}(x-y)\right)+1394\left(4^{k}\right)^{n} f\left(4^{-k n}(x+y)\right)-425\left(4^{k}\right)^{n} f\left(4^{-k n} y\right)+1530\left(4^{k}\right)^{n} f\left(4^{-k n} x\right)(t) \\
& \geq \Psi\left(4^{-k n} x, 4^{-k n} y, \frac{t}{\left|4^{k}\right|^{n}}\right) \geq \Psi\left(x, y, \frac{\alpha^{n} t}{\left|4^{k}\right|^{n}}\right)
\end{aligned}
$$

for all $x, y \in \mathcal{X}$ and all $t>0$. Since $\lim _{n \rightarrow \infty} \Psi\left(x, y, \frac{\alpha^{n} t}{\left|4^{k}\right|^{n}}\right)=1$, we infer that $Q$ is a quartic mapping. 
If $Q^{\prime}: \mathcal{X} \rightarrow \mathcal{Y}$ is another quartic mapping such that $\mu_{Q^{\prime}(x)-f(x)}(t) \geq M(x, t)$ for all $x \in \mathcal{X}$ and $t>0$, then for each $n \in N, x \in \mathcal{X}$ and $t>0$,

$$
\left.\mu_{Q(x)-Q^{\prime}(x)}(t) \geq T\left(\mu_{Q(x)-\left(4^{4 k}\right)^{n} f\left(\frac{x}{\left(4^{k}\right)^{n}}\right)}(t), \mu_{\left(4^{4 k}\right)^{n} f\left(\frac{x}{\left(4^{k}\right)^{n}}\right)-Q^{\prime}(x)}(t), t\right)\right) .
$$

Thanks to (4.8), we conclude that $Q=Q$ '.

Corollary 4.3. Let $\mathcal{K} b e$ a non-Archimedean field, $\mathcal{X}$ a vector space over $\mathcal{K}$ and let $(\mathcal{Y}, \mu, T)$ be a non-Archimedean random Banach space over Kunder a $t$-norm $T \in \mathcal{H}$. Let $f: \mathcal{X} \rightarrow \mathcal{Y}$ be a $\Psi$-approximately quartic mapping. If, for some $\alpha \in \mathbb{R}, \alpha>0$, and some integer $k, k>3$, with $\left|4^{k}\right|<\alpha$,

$$
\Psi\left(4^{-k} x, 4^{-k} y, t\right) \geq \Psi(x, y, \alpha t) \quad(x \in \mathcal{X}, \quad t>0),
$$

then there exists a unique quartic mapping $Q: \mathcal{X} \rightarrow \mathcal{Y}$ such that

$$
\mu_{f(x)-Q(x)}(t) \geq T_{i=1}^{\infty} M\left(x, \frac{\alpha^{i+1} t}{|4|^{k i}}\right)
$$

for all $x \in \mathcal{X}$ and all $t>0$, where

$$
M(x, t):=T\left(\Psi(x, 0, t), \Psi(4 x, 0, t), \cdots, \Psi\left(4^{k-1} x, 0, t\right)\right) \quad(x \in \mathcal{X}, \quad t>0) .
$$

Proof. Since

$$
\lim _{n \rightarrow \infty} M\left(x, \frac{\alpha^{j} t}{|4|^{k j}}\right)=1 \quad(x \in \mathcal{X}, \quad t>0)
$$

and $T$ is of Hadžić type, from Proposition 2.1, it follows that

$$
\lim _{n \rightarrow \infty} T_{j=n}^{\infty} M\left(x, \frac{\alpha^{j} t}{|4|^{k j}}\right)=1 \quad(x \in \mathcal{X}, \quad t>0) .
$$

Now we can apply Theorem 4.2 to obtain the result. $\square$

Example 4.4. Let $\left(\mathcal{X}, \mu, T_{M}\right)$ non-Archimedean random normed space in which

$$
\mu_{x}(t)=\frac{t}{t+\|x\|}, \quad \forall x \in \mathcal{X}, \quad t>0,
$$

and $\left(\mathcal{Y}, \mu, T_{M}\right)$ a complete non-Archimedean random normed space (see Example 3.2). Define

$$
\Psi(x, y, t)=\frac{t}{1+t} .
$$

It is easy to see that (4.2) holds for $\alpha=1$. Also, since

$$
M(x, t)=\frac{t}{1+t}
$$

we have

$$
\begin{aligned}
\lim _{n \rightarrow \infty} T_{M, j=n}^{\infty} M\left(x, \frac{\alpha^{j} t}{|4|^{k j}}\right) & =\lim _{n \rightarrow \infty}\left(\lim _{m \rightarrow \infty} T_{M, j=n}^{m} M\left(x, \frac{t}{|4|^{k j}}\right)\right) \\
& =\lim _{n \rightarrow \infty} \lim _{m \rightarrow \infty}\left(\frac{t}{t+\left|4^{k}\right|^{n}}\right) \\
& =1, \quad \forall x \in \mathcal{X}, \quad t>0 .
\end{aligned}
$$


Let $f: \mathcal{X} \rightarrow \mathcal{Y}$ be a $\Psi$-approximately quartic mapping. Thus all the conditions of Theorem 4.2 hold and so there exists a unique quartic mapping $Q: \mathcal{X} \rightarrow \mathcal{Y}$ such that

$$
\mu_{f(x)-Q(x)}(t) \geq \frac{t}{t+\left|4^{k}\right|} .
$$

\section{Fixed point method for random stability of the quartic functional equation}

In this section, we apply a fixed point method for achieving random stability of the quartic functional equation. The notion of generalized metric space has been introduced by Luxemburg [37], by allowing the value $+\infty$ for the distance mapping. The following lemma (Luxemburg-Jung theorem) will be used in the proof of Theorem 5.3.

Lemma 5.1. [38]. Let $(X, d)$ be a complete generalized metric space and let $A: X \rightarrow$ $X$ be a strict contraction with the Lipschitz constant $k$ such that $d\left(x_{0}, A\left(x_{0}\right)\right)<+\infty$ for some $x_{0} \in X$. Then $A$ has a unique fixed point in the set $Y:=\left\{y \in X, d\left(x_{0}, y\right)<\infty\right\}$ and the sequence $\left(A^{n}(x)\right)_{n \in N}$ converges to the fixed point $x^{*}$ for every $x \in Y$. Moreover, $d\left(x_{0}\right.$, $\left.A\left(x_{0}\right)\right) \leq \delta$ implies $d\left(x^{*}, x_{0}\right) \leq \frac{\delta}{1-k}$.

Let $X$ be a linear space, $\left(Y, v, T_{M}\right)$ a complete $\mathrm{RN}$-space and let $G$ be a mapping from $X \times R$ into $[0,1]$, such that $G(x,.) \in D^{+}$for all $x$. Consider the set $E:=\{g: X \rightarrow$ $Y, g(0)=0\}$ and the mapping $d_{G}$ defined on $E \times E$ by

$$
d_{G}(g, h)=\inf \left\{u \in R^{+}, v_{g(x)-h(x)}(u t) \geq G(x, t) \text { for all } x \in X \text { and } t>0\right\}
$$

where, as usual, $\inf \varnothing=+\infty$. The following lemma can be proved as in [22]:

Lemma 5.2. cf. $[22,39] d_{G}$ is a complete generalized metric on $E$.

Theorem 5.3. Let $X$ be a real linear space, $t f$ a mapping from $X$ into a complete $R N$ space $\left(Y, \mu, T_{M}\right)$ with $f(0)=0$ and let $\Phi: X^{2} \rightarrow D^{+}$be a mapping with the property

$$
\exists \alpha \in(0,256): \Phi_{4 x, 4 y}(\alpha t) \geq \Phi_{x, y}(t), \forall x, y \in X, \quad \forall t>0 .
$$

If

$$
\begin{aligned}
& \mu_{16 f(x+4 y)+f(4 x-y)-306\left[9 f\left(x+\frac{y}{3}\right)+f(x+2 y)\right]-136 f(x-y)+1394 f(x+y)-425 f(y)+1530 f(x)}(t) \\
& \geq \Phi_{x, y}(t), \quad \forall x, y \in X
\end{aligned}
$$

then there exists a unique quartic mapping $g: X \rightarrow Y$ such that

$$
\mu_{g(x)-f(x)}(t) \geq \Phi_{x, 0}(M t), \quad \forall x \in X, \quad \forall t>0,
$$

where

$$
M=(256-\alpha) .
$$

Moreover,

$$
g(x)=\lim _{n \rightarrow \infty} \frac{f\left(4^{n} x\right)}{4^{4 n}} .
$$

Proof. By setting $y=0$ in (5.2), we obtain

$$
\mu_{f(4 x)-256 f(x)}(t) \geq \Phi_{x, 0}(t)
$$


for all $x \in X$, whence

$$
\begin{aligned}
\mu \frac{1}{256} f(4 x)-f(x) & (t)=\mu \frac{1}{256}(f(4 x)-256 f(x)) \\
& =\mu_{f(4 x)-256 f(x)(256 t)} \\
& \geq \Phi_{x, 0}(256 t), \quad \forall x \in X, \quad \forall t>0 .
\end{aligned}
$$

Let

$$
G(x, t):=\Phi_{x, 0}(256 t) .
$$

Consider the set

$$
E:=\{g: X \rightarrow Y, g(0)=0\}
$$

and the mapping $d_{G}$ defined on $E \times E$ by

$$
d_{G}(g, h)=\inf \left\{u \in R^{+}, \mu_{g(x)-h(x)}(u t) \geq G(x, t) \text { for all } x \in X \text { and } t>0\right\} .
$$

By Lemma 5.2, $\left(E, d_{G}\right)$ is a complete generalized metric space. Now, let us consider the linear mapping $J: E \rightarrow E$,

$$
J g(x):=\frac{1}{256} g(4 x)
$$

We show that $J$ is a strictly contractive self-mapping of $E$ with the Lipschitz constant $k=\alpha / 256$.

Indeed, let $g, h \in E$ be mappings such that $d_{G}(g, h)<\varepsilon$. Then

$$
\mu_{g(x)-h(x)}(\varepsilon t) \geq G(x, t), \forall x \in X, \quad \forall t>0,
$$

whence

$$
\begin{aligned}
\mu_{J g(x)-J h(x)}\left(\frac{\alpha}{256} \varepsilon t\right) & =\mu \frac{1}{256}(g(4 x)-h(4 x)) \\
& =\mu_{g(4 x)-h(4 x)}(\alpha \varepsilon t) \\
& \geq G(4 x, \alpha t) \quad(x \in X, \quad t>0) .
\end{aligned}
$$

Since $G(4 x, \alpha t) \geq G(x, t), \mu_{J g(x)-J h(x)}\left(\frac{\alpha}{256} \varepsilon t\right) \geq G(x, t)$, that is,

$$
d_{G}(g, h)<\varepsilon \Rightarrow d_{G}(J g, J h) \leq \frac{\alpha}{256} \varepsilon .
$$

This means that

$$
d_{G}(J g, J h) \leq \frac{\alpha}{256} d_{G}(g, h)
$$

for all $g, h$ in $E$.

Next, from

$$
\mu_{f(x)-\frac{1}{256} f(4 x)}(t) \geq G(x, t)
$$

it follows that $d_{G}(f, \mathrm{Jf}) \leq 1$. Using the Luxemburg-Jung theorem, we deduce the existence of a fixed point of $J$, that is, the existence of a mapping $g: X \rightarrow Y$ such that $g(4 x)$ $=256 g(x)$ for all $x \in X$. 
Since, for any $x \in X$ and $t>0$,

$$
d_{G}(u, v)<\varepsilon \Rightarrow \mu_{u(x)-v(x)}(t) \geq G\left(x, \frac{t}{\varepsilon}\right),
$$

from $d_{G}\left(J^{n} f, g\right) \rightarrow 0$, it follows that $\lim _{n \rightarrow \infty} \frac{f\left(4^{n} x\right)}{4^{4 n}}=g(x)$ for any $x \in X$.

Also, $d_{G}(f, g) \leq \frac{1}{1-L} d(f, J f)$ implies the inequality $d_{G}(f, g) \leq \frac{1}{1-\frac{\alpha}{256}}$ from which it immediately follows $v_{g(x)-f(x)}\left(\frac{256}{256-\alpha} t\right) \geq G(x, t)$ for all $t>0$ and all $x \in X$. This means that

$$
\mu_{g(x)-f(x)}(t) \geq G\left(x, \frac{256-\alpha}{256} t\right), \quad \forall x \in X, \quad \forall t>0 .
$$

It follows that

$$
\mu_{g(x)-f(x)}(t) \geq \Phi_{x, 0}((256-\alpha) t) \quad \forall x \in X, \quad \forall t>0 .
$$

The uniqueness of $g$ follows from the fact that $g$ is the unique fixed point of $J$ with the property: there is $C \in(0, \infty)$ such that $\mu_{g(x)-f(x)}(C t) \geq G(x, t)$ for all $x \in X$ and all $t$ $>0$, as desired. $\square$

\section{Intuitionistic random normed spaces}

Recently, the notation of intuitionistic random normed space introduced by Chang et al. [19]. In this section, we shall adopt the usual terminology, notations, and conventions of the theory of intuitionistic random normed spaces as in [22], [31], [33], [34], [40], [41], [42].

Definition 6.1. A measure distribution function is a function $\mu: R \rightarrow[0,1]$ which is left continuous, non-decreasing on $R, \inf _{t \in R} \mu(t)=0$ and $\sup _{t \in R} \mu(t)=1$.

We will denote by $D$ the family of all measure distribution functions and by $H$ a special element of $D$ defined by

$$
H(t)=\left\{\begin{array}{l}
0, \text { if } t \leq 0 \\
1, \text { if } t>0
\end{array}\right.
$$

If $X$ is a nonempty set, then $\mu: X \rightarrow D$ is called a probabilistic measure on $X$ and $\mu$ $(x)$ is

denoted by $\mu_{x}$.

Definition 6.2. A non-measure distribution function is a function $v: R \rightarrow[0,1]$ which is right continuous, non-increasing on $R, \inf _{t \in R} v(t)=0$ and $\sup _{t \in R} v(t)=1$.

We will denote by $B$ the family of all non-measure distribution functions and by $G$ a special element of $B$ defined by

$$
G(t)=\left(\begin{array}{l}
1, \text { if } t \leq 0 \\
0, \text { if } t>0
\end{array}\right.
$$

If $X$ is a nonempty set, then $v: X \rightarrow B$ is called a probabilistic non-measure on $X$ and $v(x)$ is denoted by $v_{x}$.

Lemma 6.3. [43], [44]Consider the set $L^{*}$ and operation $\leq_{L^{*}}$ defined by:

$$
\begin{gathered}
L^{*}=\left\{\left(x_{1}, x_{2}\right):\left(x_{1}, x_{2}\right) \in[0,1]^{2} \text { and } x_{1}+x_{2} \leq 1\right\}, \\
\left(x_{1}, x_{2}\right) \leq_{L *}\left(y_{1}, y_{2}\right) \Leftrightarrow x_{1} \leq y_{1}, x_{2} \geq y_{2}, \quad \forall\left(x_{1}, x_{2}\right),\left(y_{1}, y_{2}\right) \in L^{*} .
\end{gathered}
$$


Then $\left(L^{*}, \leq_{L^{*}}\right)$ is a complete lattice.

We denote its units by $0_{L^{*}}=(0,1)$ and $1_{L^{*}}=(1,0)$. In Section 2, we presented classical $t$-norm. Using the lattice $\left(L^{*}, \leq_{L^{*}}\right)$, these definitions can be straightforwardly extended.

Definition 6.4. [44] A triangular norm ( $t$-norm) on $L^{*}$ is a mapping $\mathcal{T}:\left(L^{*}\right)^{2} \rightarrow L^{*}$ satisfying the following conditions:

(a) $\left(\forall x \in L^{*}\right)\left(\mathcal{T}\left(x, 1_{L^{*}}\right)=x\right)$ (boundary condition);

(b) $\left(\forall(x, y) \in\left(L^{*}\right)^{2}\right)(\mathcal{T}(x, y)=\mathcal{T}(y, x))$ (commutativity);

(c) $\left(\forall(x, y, z) \in\left(L^{*}\right)^{3}\right)(\mathcal{T}(x, \mathcal{T}(y, z))=\mathcal{T}(\mathcal{T}(x, y), z))$ (associativity);

(d) $\left(\forall\left(x, x^{\prime}, y, y^{\prime}\right) \in\left(L^{*}\right)^{4}\right)\left(x \leq_{L^{*}} x^{\prime}\right.$ and $\left.y \leq_{L *} y^{\prime} \Rightarrow \mathcal{T}(x, y) \leq_{L^{*}} \mathcal{T}\left(x^{\prime}, y^{\prime}\right)\right)$ (monotonicity).

If $\left(L^{*}, \leq_{L^{*}}, \mathcal{T}\right)$ is an Abelian topological monoid with unit $1_{L^{*}}$, then $\mathcal{T}$ is said to be a continuous t-norm.

Definition 6.5. [44] A continuous $t$-norm $\mathcal{T}$ on $L^{*}$ is said to be continuous t-representable if there exist a continuous $t$-norm " and a continuous $t$-conorm $\diamond$ on $[0,1]$ such that, for all $x=\left(x_{1}, x_{2}\right), y=\left(y_{1}, y_{2}\right) \in L^{*}$,

$$
\mathcal{T}(x, y)=\left(x_{1} * y_{1}, x_{2} \diamond y_{2}\right) .
$$

For example,

$$
\mathcal{T}(a, b)=\left(a_{1} b_{1}, \min \left\{a_{2}+b_{2}, 1\right\}\right)
$$

and

$$
\mathbf{M}(a, b)=\left(\min \left\{a_{1}, b_{1}\right\}, \max \left\{a_{2}, b_{2}\right\}\right)
$$

are continuous $t$-representable for all $a=\left(a_{1}, a_{2}\right), b=\left(b_{1}, b_{2}\right) \in L^{*}$.

Now, we define a sequence $\mathcal{T}^{n}$ recursively by $\mathcal{T}^{1}=\mathcal{T}$ and

$$
\mathcal{T}^{n}\left(x^{(1)}, \ldots, x^{(n+1)}\right)=\mathcal{T}\left(\mathcal{T}^{n-1}\left(x^{(1)}, \ldots, x^{(n)}\right), x^{(n+1)}\right), \quad \forall n \geq 2, \quad x^{(i)} \in L^{*} .
$$

Definition 6.6. A negator on $L^{*}$ is any decreasing mapping $\mathcal{N}: L^{*} \rightarrow L^{*}$ satisfying $\mathcal{N}\left(1_{L^{*}}\right)=0_{L^{*}}$ and $\mathcal{N}\left(1_{L^{*}}\right)=0_{L^{*}}$. If $\mathcal{N}(\mathcal{N}(x))=x$ for all $x \in L^{*}$, then $\mathcal{N}$ is called an involutive negator. A negator on $[0,1]$ is a decreasing function $N:[0,1] \rightarrow[0,1]$ satisfying $N(0)=1$ and $N(1)=0 . N_{s}$ denotes the standard negator on $[0,1]$ defined by

$$
N_{s}(x)=1-x, \quad \forall x \in[0,1] .
$$

Definition 6.7. Let $\mu$ and $v$ be measure and non-measure distribution functions from $X \times(0,+\infty)$ to $[0,1]$ such that $\mu_{x}(t)+v_{x}(t) \leq 1$ for all $x \in X$ and $t>0$. The triple $\left(X, \mathcal{P}_{\mu, v}, \mathcal{T}\right)$ is said to be an intuitionistic random normed space (briefly IRN-space) if $X$ is a vector space, $\mathcal{T}$ is continuous $t$-representable and $\mathcal{P}_{\mu, v}$ is a mapping $X \times(0,+\infty) \rightarrow$ $L^{*}$ satisfying the following conditions: for all $x, y \in X$ and $t, s>0$,
(a) $\mathcal{P}_{\mu, v}(x, 0)=0_{L^{*}}$
(b) $\mathcal{P}_{\mu, v}(x, t)=1_{L^{*}}$ if and only if $x=0$;
(c) $\mathcal{P}_{\mu, \nu}(\alpha x, t)=\mathcal{P}_{\mu, \nu}\left(x, \frac{t}{|\alpha|}\right)$ for all $\alpha \neq 0$;
(d) $\mathcal{P}_{\mu, v}(x+y, t+s) \geq_{L^{*}} \mathcal{T}\left(\mathcal{P}_{\mu, v}(x, t), \mathcal{P}_{\mu, v}(y, s)\right)$ 
In this case, $\mathcal{P}_{\mu, v}$ is called an intuitionistic random norm. Here,

$$
\mathcal{P}_{\mu, v}(x, t)=\left(\mu_{x}(t), v_{x}(t)\right) \text {. }
$$

Example 6.8. Let $(X,\|\cdot\|)$ be a normed space. Let $\mathcal{T}(a, b)=\left(a_{1} b_{1}, \min \left(a_{2}+b_{2}, 1\right)\right)$ for all $a=\left(a_{1}, a_{2}\right), b=\left(b_{1}, b_{2}\right) \in L^{*}$ and let $\mu, v$ be measure and non-measure distribution functions defined by

$$
\mathcal{P}_{\mu, v}(x, t)=\left(\mu_{x}(t), v_{x}(t)\right)=\left(\frac{t}{t+\|x\|}, \frac{\|x\|}{t+\|x\|}\right), \quad \forall t \in R^{+} .
$$

Then $\left(X, \mathcal{P}_{\mu, \nu}, \mathcal{T}\right)$ is an IRN-space.

Definition 6.9. (1) A sequence $\left\{x_{n}\right\}$ in an IRN-space $\left(X, \mathcal{P}_{\mu, v}, \mathcal{T}\right)$ is called a Cauchy sequence if, for any $\varepsilon>0$ and $t>0$, there exists an $n_{0} \in \mathbb{N}$ such that

$$
\mathcal{P}_{\mu, v}\left(x_{n}-x_{m}, t\right)>_{L^{*}}\left(N_{s}(\varepsilon), \varepsilon\right), \quad \forall n, m \geq n_{0},
$$

where $N_{s}$ is the standard negator.

(2) The sequence $\left\{x_{n}\right\}$ is said to be convergent to a point $x \in X$ (denoted by $x_{n} \stackrel{\mathcal{P}_{\mu, v}}{\rightarrow} x$ ) if $\mathcal{P}_{\mu, v}\left(x_{n}-x, t\right) \rightarrow 1_{L^{*}}$ as $n \rightarrow \infty$ for every $t>0$.

(3) An IRN-space $\left(X, \mathcal{P}_{\mu, v}, \mathcal{T}\right)$ is said to be complete if every Cauchy sequence in $X$ is convergent to a point $x \in X$.

\section{Stability results in intuitionistic random normed spaces}

In this section, we prove the generalized Ulam-Hyers stability of the quartic functional equation in intuitionistic random normed spaces.

Theorem 7.1. Let $X$ be a linear space and let $\left(X, \mathcal{P}_{\mu, v}, \mathcal{T}\right)$ be a complete IRN-space. Let $f: X \rightarrow Y$ be a mapping with $f(0)=0$ for which there are $\xi, \zeta: X^{2} \rightarrow D^{+}$, where $\xi$ $(x, y)$ is denoted by $\xi_{x, y}$ and $\zeta_{(x, y)}$ is denoted by $\zeta_{x, y}$, further, $\left(\xi_{x, y}(t), \zeta_{x, y}(t)\right)$ is denoted by $Q_{\xi, \zeta}(x, y, t)$, with the property:

$$
\begin{gathered}
\mathcal{P}_{\mu, \nu}\left(16 f(x+4 y)+f(4 x-y)-306\left[9 f\left(x+\frac{y}{3}\right)+f(x+2 y)\right]\right. \\
-136 f(x-y)+1394 f(x+y)-425 f(y)+1530 f(x), t) \\
\geq_{L^{*}} Q_{\xi, \zeta}(x, y, t) .
\end{gathered}
$$

If

$$
\mathcal{T}_{i=1}^{\infty}\left(Q_{\xi, \zeta}\left(4^{n+i-1} x, 0,4^{4 n+3 i+3} t\right)\right)=1_{L^{*}}
$$

and

$$
\lim _{n \rightarrow \infty} Q_{\xi, \zeta}\left(4^{n} x, 4^{n} y, 4^{4 n} t\right)=1_{L^{*}}
$$

for all $x, y \in X$ and all $t>0$, then there exists a unique quartic mapping $Q: X \rightarrow Y$ such that

$$
\mathcal{P}_{\mu, v}(f(x)-Q(x), t) \geq_{L^{*}} \mathcal{T}_{i=1}^{\infty}\left(Q_{\xi, \zeta}\left(4^{i-1} x, 0,4^{3 i+3} t\right)\right)
$$

Proof. Putting $y=0$ in (7.1), we have

$$
\mathcal{P}_{\mu, v}\left(\frac{f(4 x)}{256}-f(x), t\right) \geq_{L^{*}} Q_{\xi, \zeta}\left(x, 0,4^{4} t\right) .
$$


Therefore, it follows that

$$
\mathcal{P}_{\mu, \nu}\left(\frac{f\left(4^{k+1} x\right)}{4^{4(k+1)}}-\frac{f\left(4^{k} x\right)}{4^{4 k}}, \frac{t}{4^{4 k}}\right) \geq_{L^{*}} Q_{\xi, \zeta}\left(4^{k} x, 0,4^{4} t\right),
$$

which implies that

$$
\mathcal{P}_{\mu, v}\left(\frac{f\left(4^{k+1} x\right)}{4^{4(k+1)}}-\frac{f\left(4^{k} x\right)}{4^{4 k}}, t\right) \geq_{L^{*}} Q_{\xi, \zeta}\left(4^{k} x, 0,4^{4(k+1)} t\right),
$$

that is,

$$
\mathcal{P}_{\mu, \nu}\left(\frac{f\left(4^{k+1} x\right)}{4^{4(k+1)}}-\frac{f\left(4^{k} x\right)}{4^{4 k}}, \frac{t}{4^{k+1}}\right) \geq_{L *} Q_{\xi, \zeta}\left(4^{k} x, 0,4^{4(k+1)} t\right)
$$

for all $k \in N$ and all $t>0$. As $1>1 / 4+\ldots+1 / 4^{n}$, from the triangle inequality, it follows

$$
\begin{aligned}
\mathcal{P}_{\mu, v}\left(\frac{f\left(4^{n} x\right)}{256^{n}}-f(x), t\right) & \geq_{L^{*}} \mathcal{T}_{k=0}^{n-1}\left(\mathcal{P}_{\mu, \nu}\left(\frac{f\left(4^{k+1} x\right)}{4^{4(k+1)}}-\frac{f\left(4^{k} x\right)}{4^{4 k}}, \sum_{k=0}^{n-1} \frac{1}{4^{k+1}} t\right)\right) \\
& \geq_{L^{*}} \mathcal{T}_{i=1}^{n}\left(Q_{\xi, \zeta}\left(4^{i-1} x, 0,4^{3 i+3} t\right)\right) .
\end{aligned}
$$

In order to prove convergence of the sequence $\left\{\frac{f\left(4^{n} x\right)}{256^{n}}\right\}$, replacing $x$ with $4^{m} x$ in (7.9), we get that for $m, n>0$

$$
\mathcal{P}_{\mu, v}\left(\frac{f\left(4^{n+m} x\right)}{256^{(n+m)}}-\frac{f\left(4^{m} x\right)}{256^{m}}, t\right) \geq_{L^{*}} \mathcal{T}_{i=1}^{n}\left(Q_{\xi, \zeta}\left(4^{i+m-1} x, 0,4^{3 i+4 m+3} t\right)\right) .
$$

Since the right-hand side of the inequality tends $1_{L^{*}}$ as $m$ tends to infinity, the sequence $\left\{\frac{f\left(4^{n} x\right)}{4^{4 n}}\right\}$ is a Cauchy sequence. So we may define $Q(x)=\lim _{n \rightarrow \infty} \frac{f\left(4^{n} x\right)}{4^{4 n}}$ for all $x \in X$.

Now, we show that $Q$ is a quartic mapping. Replacing $x, y$ with $4^{n} x$ and $4^{n} y$, respectively, in (7.1), we obtain

$$
\begin{aligned}
& \mathcal{P}_{\mu, v} \frac{f\left(4^{n}(x+4 y)\right)}{256^{n}}+\frac{f\left(4^{n}(4 x-y)\right)}{256^{n}}-\frac{306\left[9 f\left(4^{n}\left(x+\frac{y}{3}\right)\right)+f\left(4^{n}(x+2 y)\right)\right.}{256^{n}} \\
&\left.\quad-\frac{136 f\left(4^{n}(x-y)\right)}{256^{n}}+\frac{1394 f\left(4^{n}(x+y)\right)}{256^{n}}-\frac{425 f\left(4^{n}(y)\right)}{256^{n}}+\frac{1530 f\left(4^{n}(x)\right)}{256^{n}}, t\right) \\
& \quad \geq_{L *} Q_{\xi, \zeta}\left(4^{n} x, 4^{n} y, 4^{4 n} t\right) .
\end{aligned}
$$

Taking the limit as $n \rightarrow \infty$, we find that $Q$ satisfies (1.1) for all $x, y \in X$.

Taking the limit as $n \rightarrow \infty$ in (7.9), we obtain (7.4).

To prove the uniqueness of the quartic mapping $Q$ subject to (7.4), let us assume that there exists another quartic mapping $Q$ ' which satisfies (7.4). Obviously, we have $x$ $\in X$ and all $n \in \mathbb{N}$. Hence it follows from (7.4) that

$$
\begin{aligned}
& \mathcal{P}_{\mu, \nu}\left(Q(x)-Q^{\prime}(x), t\right) \\
& \quad \geq_{L^{*}} \mathcal{P}_{\mu, v}\left(Q\left(4^{n} x\right)-Q^{\prime}\left(4^{n} x\right), 4^{4 n} t\right) \\
& \quad \geq_{L^{*}} \mathcal{T}\left(\mathcal{P}_{\mu, v}\left(Q\left(4^{n} x\right)-f\left(4^{n} x\right), 4^{4 n-1} t\right), \mathcal{P}_{\mu, \nu}\left(f\left(4^{n} x\right)-Q^{\prime}\left(4^{n} x\right), 4^{4 n-1} t\right)\right) \\
& \quad \geq_{L^{*}} \mathcal{T}\left(\mathcal{T}_{i=1}^{\infty}\left(Q_{\xi, \zeta}\left(4^{n+i-1} x, 0,4^{4 n+3 i+3} t\right)\right), \mathcal{T}_{i=1}^{\infty}\left(Q_{\xi, \zeta}\left(4^{n+i-1} x, 0,4^{4 n+3 i+3} t\right)\right)\right.
\end{aligned}
$$


for all $x \in X$. By letting $n \rightarrow \infty$ in (7.4), we prove the uniqueness of $Q$. This completes the proof of the uniqueness, as desired.

Corollary 7.2. Let $\left(X, \mathcal{P}^{\prime}{ }_{\mu^{\prime}, v^{\prime}}, \mathcal{T}\right)$ be an IRN-space and let $\left(Y, \mathcal{P}_{\mu, \nu}, \mathcal{T}\right)$ be a complete IRN-space. Let $f: X \rightarrow Y$ be a mapping such that

$$
\begin{gathered}
\mathcal{P}_{\mu, v}\left(16 f(x+4 y)+f(4 x-y)-306\left[9 f\left(x+\frac{y}{3}\right)+f(x+2 y)\right]\right. \\
-136 f(x-y)+1394 f(x+y)-425 f(y)+1530 f(x), t) \\
\geq_{L *} \mathcal{P}^{\prime}{ }^{\prime}, \nu^{\prime} \\
(x+y, t)
\end{gathered}
$$

for all $t>0$ in which

$$
\lim _{n \rightarrow \infty} \mathcal{T}_{i=1}^{\infty}\left(\mathcal{P}_{\mu^{\prime}, v^{\prime}}^{\prime}\left(x, 4^{4 n+3 i+3} t\right)\right)=1_{L^{*}}
$$

for all $x, y \in X$. Then there exists a unique quartic mapping $Q: X \rightarrow Y$ such that

$$
\mathcal{P}_{\mu, v}(f(x)-Q(x), t) \geq_{L *} \mathcal{T}_{i=1}^{\infty}\left(\mathcal{P}_{\mu^{\prime}, v^{\prime}}^{\prime}\left(x, 4^{3 i+3} t\right)\right)
$$

Now, we give an example to illustrate the main result of Theorem 7.1 as follows.

Example 7.3. Let $(X,\|\|$.$) be a Banach algebra, \left(X, \mathcal{P}_{\mu, \nu}, \mathbf{M}\right)$ an IRN-space in which

$$
\mathcal{P}_{\mu, v}(x, t)=\left(\frac{t}{t+\|x\|}, \frac{\|x\|}{t+\|x\|}\right)
$$

and let $\left(Y, \mathcal{P}_{\mu, \nu}, \mathrm{M}\right)$ be a complete IRN-space for all $x \in X$. Define $f: X \rightarrow X$ by $f(x)$ $=x^{4}+x_{0}$, where $x_{0}$ is a unit vector in $X$. A straightforward computation shows that

$$
\begin{gathered}
\mathcal{P}_{\mu, v}\left(16 f(x+4 y)+f(4 x-y)-306\left[9 f\left(x+\frac{y}{3}\right)+f(x+2 y)\right]\right. \\
-136 f(x-y)+1394 f(x+y)-425 f(y)+1530 f(x), t) \\
\geq_{L *} \mathcal{P}_{\mu, v}(x+y, t), \quad \forall t>0 .
\end{gathered}
$$

Also

$$
\begin{aligned}
\lim _{n \rightarrow \infty} M_{i=1}^{\infty}\left(\mathcal{P}_{\mu, v}\left(4^{n+i-1} x, 4^{4 n+3 i+3} t\right)\right) & =\lim _{n \rightarrow \infty} \lim _{m \rightarrow \infty} M_{i=1}^{m}\left(\mathcal{P}_{\mu, v}\left(x, 4^{3 n+2 i+4} t\right)\right) \\
& =\lim _{n \rightarrow \infty} \lim _{m \rightarrow \infty} \mathcal{P}_{\mu, v}\left(x, 4^{3 n+6} t\right) \\
& =\lim _{n \rightarrow \infty} \mathcal{P}_{\mu, v}\left(x, 4^{3 n+6} t\right) \\
& =1_{L^{*}} .
\end{aligned}
$$

Therefore, all the conditions of 7.1 hold and so there exists a unique quartic mapping $Q: X \rightarrow Y$ such that

$$
\mathcal{P}_{\mu, v}(f(x)-Q(x), t) \geq_{L^{*}} \mathcal{P}_{\mu, v}\left(x, 4^{6} t\right) .
$$

\section{Author details}

${ }^{1}$ Section of Mathematics and Informatics, Pedagogical Department, National and Capodistrian University of Athens, 4 , Agamemnonos St., Aghia Paraskevi, Athens 15342, Greece ²Department of Mathematics, Science and Research Branch, Islamic Azad University, Tehran, Iran ${ }^{3}$ Faculty of Mathematics and Computer Sciences, Sabzevar Tarbiat Moallem University, Sabzevar, Iran ${ }^{4}$ Department of Mathematics, lust, Behshar, Iran 


\section{Competing interests}

The authors declare that they have no competing interests.

Received: 15 February 2011 Accepted: 18 September 2011 Published: 18 September 2011

\section{References}

1. Ulam, SM: Problems in Modern Mathematics. In Science Editions, vol. Chapter VI,Wiley, New York (1964)

2. Hyers, DH: On the stability of the linear functional equation. Proc Natl Acad Sci USA. 27, $222-224$ (1941). doi:10.1073/ pnas.27.4.222

3. Aoki, T: On the stability of the linear transformation in Banach spaces. J Math Soc Jpn. 2, 64-66 (1950). doi:10.2969/ jmsj/00210064

4. Rassias, ThM: On the stability of the linear mapping in Banach spaces. Proc Am Math Soc. 72, 297-300 (1978). doi:10.1090/50002-9939-1978-0507327-1

5. Baak, C, Moslehian, MS: On the stability of ${ }^{*}$-homomorphisms. Nonlinear Anal TMA. 63, $42-48$ (2005). doi:10.1016/j. na.2005.04.004

6. Chudziak, J, Tabor, J: Generalized Pexider equation on a restricted domain. J Math Psychol. 52, 389-392 (2008). doi:10.1016/j.jmp.2008.04.002

7. Czerwik, S: Functional Equations and Inequalities in Several Variables. World Scientific, River Edge, NJ. (2002)

8. Eshaghi Gordji, M, Rassias, JM, Savakohi, MB: Approximation of the quadratic and cubic functional equations in RNspaces. Eur J Pure Appl Math. 2(4), 494-507 (2009)

9. Hyers, DH, Isac, G, Rassias, ThM: Stability of Functional Equations in Several Variables. Birkhäuser, Basel. (1998)

10. Jung, S: Hyers-Ulam-Rassias Stability of Functional Equations in Mathematical Analysis. Hadronic Press, Palm Harbor (2001)

11. Rassias, JM: On approximation of approximately linear mappings by linear mappings. J Funct Anal. 46, 126-130 (1982). doi:10.1016/0022-1236(82)90048-9

12. Rassias, JM: On approximation of approximately linear mappings by linear mappings. Bull Sci Math. 108, 445-446 (1984)

13. Rassias, JM: Solution of a problem of Ulam. J Approx Theory. 57, 268-273 (1989). doi:10.1016/0021-9045(89)90041-5

14. Rassias, JM: Solution of the Ulam stability problem for the quartic mapping. Glasnik Matematicki. 34(54), 243-252 (1999)

15. Rassias, ThM: On the stability of functional equations and a problem of Ulam. Acta Appl Math. 62, 23-130 (2000). doi:10.1023/A:1006499223572

16. Rassias, ThM: Functional Equations, Inequalities and Applications. Kluwer Academic Publishers, Dordrecht (2003)

17. Ravi, K, Rassias, JM, Arunkumar, $M$, Kodandan, R: Stability of a generalized mixed type additive, quadratic, cubic and quartic functional equation. JPAM 10(4), 29 (2009). Article ID 114

18. Alsina, C: On the stability of a functional equation arising in probabilistic normed spaces. General Inequalities, Oberwolfach 5, 263-271 (1986). Birkh?ä?user, Basel (1987)

19. Chang, SS, Rassias, JM, Saadati, R: The stability of the cubic functional equation in intuitionistic random normed spaces. Appl Math Mech. 31, 21-26 (2010). doi:10.1007/s10483-010-0103-6

20. Mirmostafaee, M, Mirzavaziri, M, Moslehian, MS: Fuzzy stability of the Jensen functional equation. Fuzzy Set Syst. 159, 730-738 (2008). doi:10.1016/j.fss.2007.07.011

21. Mirzavaziri, M, Moslehian, MS: A fixed point approach to stability of a quadratic equation. Bull Braz Math Soc. 37, 361-376 (2006). doi:10.1007/s00574-006-0016-z

22. Miheț, $\mathrm{D}, \mathrm{Radu}, \mathrm{V}$ : On the stability of the additive Cauchy functional equation in random normed spaces. J Math Anal Appl. 343, 567-572 (2008)

23. Mihet, D: The probabilistic stability for a functional equation in a single variable. Acta Math Hungar. 123, 249-256 (2009). doi:10.1007/s10474-008-8101-y

24. Mihet, D: The fixed point method for fuzzy stability of the Jensen functional equation. Fuzzy Set Syst. 160, 1663-1667 (2009). doi:10.1016/j.fss.2008.06.014

25. Mihet, $D$, Saadati, R, Vaezpour, SM: The stability of the quartic functional equation in random normed spaces. Acta Appl Math. 110, 797-803 (2010). doi:10.1007/s10440-009-9476-7

26. Mihet, $D$, Saadati, R, Vaezpour, SM: The stability of an additive functional equation in Menger probabilistic $\varphi$-normed spaces. Math Slovaca. 61, 817-826 (2011). doi:10.2478/s12175-011-0049-7

27. Baktash, E, Cho, Y, Jalili, M, Saadati, R, Vaezpour, SM: On the stability of cubic mappings and quadratic mappings in random normed spaces. J Inequal Appl. 2008, Article ID 902187 (2008)

28. Eshaghi Gordji, M, Zolfaghari, S, Rassias, JM, Savadkouhi, MB: Solution and stability of a mixed type cubic and quartic functional equation in quasi-Banach spaces. Abst Appl Anal 2009، 14 (2009). Article ID 417473

29. Saadati, R, Vaezpour, SM, Cho, Y: A note on the "On the stability of cubic mappings and quadratic mappings in random normed spaces". J Inequal Appl. 2009, Article ID 214530 (2009)

30. Mohamadi, M, Cho, Y, Park, C, Vetro, P, Saadati, R: Random stability of an additive-quadratic-quartic functional equation. J Inequal Appl 2010, 18 (2010). Article ID 754210

31. Hadžcí, O, Pap, E: Fixed Point Theory in PM-Spaces. Kluwer Academic, Dordrecht (2001)

32. Hadžić, O, Pap, E, Budincević, M: Countable extension of triangular norms and their applications to the fixed point theory in probabilistic metric spaces. Kybernetica. 38, 363-381 (2002)

33. Šerstnev, AN: On the notion of a random normed space. Dokl Akad Nauk SSSR 149, 280-283 (1963). (in Russian)

34. Schweizer, B, Sklar, A: Probabilistic Metric Spaces. Elsevier, North Holand (1983)

35. Hensel, K: Uber eine neue Begrundung der Theorie der algebraischen Zahlen. Jahres Deutsch Math Verein. 6, 83-88 (1897)

36. Mirmostafaee, M, Moslehian, MS: Fuzzy stability of additive mappings in non-Archimedean Fuzzy normed spaces. Fuzzy Set Syst. 160, 1643-1652 (2009). doi:10.1016/j.fss.2008.10.011

37. Luxemburg, WAJ: On the convergence of successive approximations in the theory of ordinary differential equations, II. Nederl. Akad. Wetensch. Proc. Ser. A 61 = Indag. Math. 20, 540-546 (1958) 
38. Jung, C: On generalized complete metric spaces. Bull Am Math Soc. 75, 113-116 (1969). doi:10.1090/S0002-9904-196912165-8

39. Miheț, D: The stability of the additive Cauchy functional equation in non-Archimedean fuzzy normed spaces. Fuzzy Set Syst. 161, 2206-2212 (2010). doi:10.1016/j.fss.2010.02.010

40. Chang, SS, Cho, Y, Kang, Y: Nonlinear Operator Theory in Probabilistic Metric Spaces. Nova Science Publishers Inc., New York (2001)

41. Kutukcu, S, Tuna, A, Yakut, AT: Generalized contraction mapping principle in intuitionistic Menger spaces and application to differential equations. Appl Math Mech. 28, 799-809 (2007). doi:10.1007/s10483-007-0610-z

42. Saadati, R, Park, J: On the intuitionistic fuzzy topological spaces. Chaos Soliton Fract. 27, 331-344 (2006). doi:10.1016/j. chaos.2005.03.019

43. Atanassov, KT: Intuitionistic fuzzy sets. Fuzzy Set Syst. 20, 87-96 (1986). doi:10.1016/S0165-0114(86)80034-3

44. Deschrijver, G, Kerre, EE: On the relationship between some extensions of fuzzy set theory. Fuzzy Set Syst. 23, 227-235 (2003)

doi:10.1186/1029-242X-2011-62

Cite this article as: Rassias et al:: On nonlinear stability in various random normed spaces. Journal of Inequalities and Applications 2011 2011:62.

\section{Submit your manuscript to a SpringerOpen ${ }^{\circ}$} journal and benefit from:

- Convenient online submission

- Rigorous peer review

- Immediate publication on acceptance

- Open access: articles freely available online

- High visibility within the field

- Retaining the copyright to your article

Submit your next manuscript at $\boldsymbol{\nabla}$ springeropen.com 